\title{
Effectiveness of COVID-19 vaccines against symptomatic SARS-CoV-2 infection and severe outcomes with variants of concern in Ontario
}

\author{
Sharifa Nasreen 101,2, Hannah Chung1, Siyi He1, Kevin A. Brown ${ }^{1,2,3}$, Jonathan B. Gubbay ${ }^{3}$, \\ Sarah A. Buchan 1,2,3,4, Deshayne B. Fell1,5,6, Peter C. Austin ${ }^{1,7}$, Kevin L. Schwartz ${ }^{1,2,3}$, \\ Maria E. Sundaram ${ }^{1,2,8}$, Andrew Calzavara', Branson Chen ${ }^{10}{ }^{1}$, Mina Tadrous ${ }^{1,9}$, Kumanan Wilson ${ }^{10,11}$, \\ Sarah E. Wilson ${ }^{1,2,3,4}$, Jeffrey C. Kwong ${ }^{1,2,3,4,12,13 \bowtie}$ and on behalf of the Canadian Immunization \\ Research Network (CIRN) Provincial Collaborative Network (PCN) Investigators
}

SARS-CoV-2 variants of concern (VOC) are more transmissible and may have the potential for increased disease severity and decreased vaccine effectiveness. We estimated the effectiveness of BNT162b2 (Pfizer-BioNTech Comirnaty), mRNA1273 (Moderna Spikevax) and ChAdOx1 (AstraZeneca Vaxzevria) vaccines against symptomatic SARS-CoV-2 infection and COVID-19 hospitalization or death caused by the Alpha (B.1.1.7), Beta (B.1.351), Gamma (P.1) and Delta (B.1.617.2) VOC in Ontario, Canada, using a test-negative design study. We identified 682,071 symptomatic community-dwelling individuals who were tested for SARS-CoV-2, and 15,269 individuals with a COVID-19 hospitalization or death. Effectiveness against symptomatic infection $\geq 7 \mathrm{~d}$ after two doses was $89-92 \%$ against Alpha, $87 \%$ against Beta, $88 \%$ against Gamma, 82-89\% against Beta/Gamma and $87-95 \%$ against Delta across vaccine products. The corresponding estimates $\geq 14 \mathrm{~d}$ after one dose were lower. Effectiveness estimates against hospitalization or death were similar to or higher than against symptomatic infection. Effectiveness against symptomatic infection was generally lower for older adults ( $\geq 60$ years) than for younger adults ( $<60$ years) for most of the VOC-vaccine combinations. Our findings suggest that jurisdictions facing vaccine supply constraints may benefit from delaying the second dose in younger individuals to more rapidly achieve greater overall population protection; however, older adults would likely benefit most from minimizing the delay in receiving the second dose to achieve adequate protection against VOC.

volution of the SARS-CoV-2 virus over the course of the COVID-19 pandemic has resulted in the emergence of four variants of concern (VOC)-Alpha (B.1.1.7), Beta (B.1.351), Gamma (P.1) and Delta (B.1.617.2) - to date. Alpha was first detected in the United Kingdom in September 2020, Beta in South Africa in May 2020, Gamma in Brazil in November 2020 and Delta in India in October $2020^{1}$. As of 28 September 2021, 193, 142, 96 and 187 countries, territories and areas globally have reported Alpha, Beta, Gamma and Delta cases, respectively ${ }^{2}$. SARS-CoV-2 VOC are more transmissible and have the potential to cause increased disease severity and to decrease COVID-19 vaccine effectiveness ${ }^{1}$. As of the time of writing, Delta is the dominant VOC that is driving increases in infections, even in populations with high levels of access to vaccines.

Evidence of the efficacy of COVID-19 vaccines against VOC from randomized clinical trials is mostly limited to post-hoc estimates of vaccine efficacy against symptomatic infection; however, these analyses suffer from insufficient statistical power and may not have variant information for all cases. Vaccine efficacy against symptomatic infection $>14 \mathrm{~d}$ after the second dose was reported to be $70 \%$ (95\% confidence interval (CI): $44-85 \%)^{3}$ against Alpha and $10 \%(95 \% \mathrm{CI},-77 \text { to } 55 \%)^{4}$ against Beta for ChAdOx1 (AstraZeneca Vaxzevria). Efficacy against infection by Beta $>7 \mathrm{~d}$ after the second dose for BNT162b2 (Pfizer-BioNTech Comirnaty) was $100 \%$ $(95 \% \text { CI, 54-100\%) })^{5}$. Few observational studies have reported the effectiveness of COVID-19 vaccines against infection or severe outcomes caused by $\mathrm{VOC}^{6-11}$.

Ontario, the largest province in Canada, implemented a three-phased COVID-19 vaccination programme starting in December 2020 and adopted a delayed second-dose strategy due to vaccine supply constraints during phase 1 (ref. ${ }^{12}$ ). BNT162b2 became available on 14 December 2020, mRNA-1273 (Moderna Spikevax) on 28 December 2020 and ChAdOx1 on 10 March $2021^{13}$. All four VOC have been circulating at various times in Ontario in $2021^{14}$. Our objective was to estimate the effectiveness of BNT162b2, mRNA-1273 and ChAdOx1 vaccines against symptomatic SARS-CoV-2 infection and severe outcomes (COVID-19 hospitalization or death) caused by Alpha, Beta, Gamma and Delta between December 2020 and August 2021 in Ontario.

\footnotetext{
IICES, Toronto, Ontario, Canada. ${ }^{2}$ Dalla Lana School of Public Health, University of Toronto, Toronto, Ontario, Canada. ${ }^{3}$ Public Health Ontario, Toronto, Ontario, Canada. ${ }^{4}$ Centre for Vaccine Preventable Diseases, University of Toronto, Toronto, Ontario, Canada. ${ }^{5}$ School of Epidemiology and Public Health, University of Ottawa, Ottawa, Ontario, Canada. ${ }^{6}$ Children's Hospital of Eastern Ontario Research Institute, Ottawa, Ontario, Canada. ${ }^{7}$ Institute of Health Policy, Management and Evaluation, University of Toronto, Toronto, Ontario, Canada. ${ }^{8}$ Center for Clinical Epidemiology and Population Health, Marshfield Clinic Research Institute, Marshfield, WI, USA. ' Women's College Hospital, Toronto, Ontario, Canada. ${ }^{10}$ Department of Medicine, University of Ottawa, Ottawa, Ontario, Canada. "Bruyere and Ottawa Hospital Research Institutes, Ottawa, Ontario, Canada. ${ }^{2}$ Department of Family and Community Medicine,

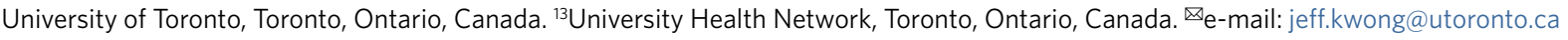




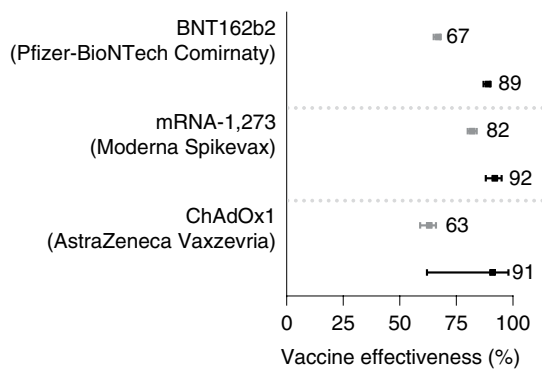

d

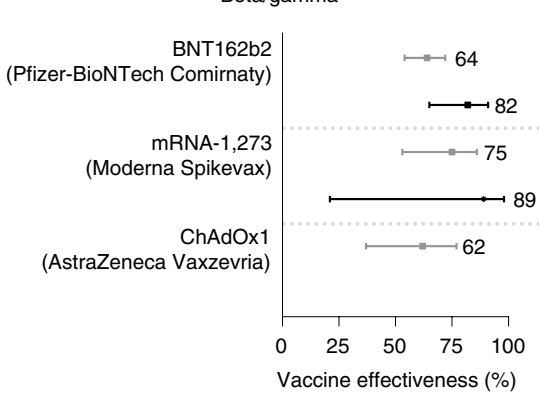

b

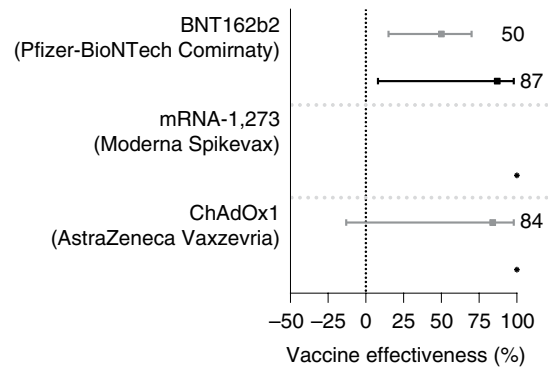

e

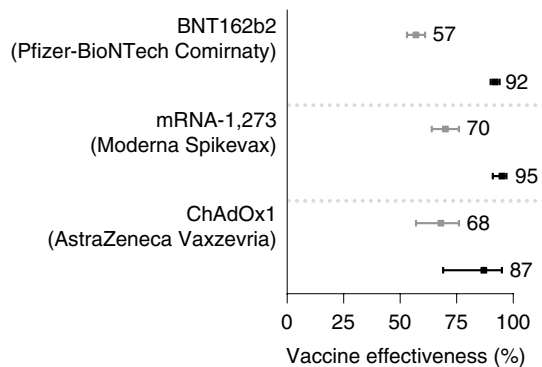

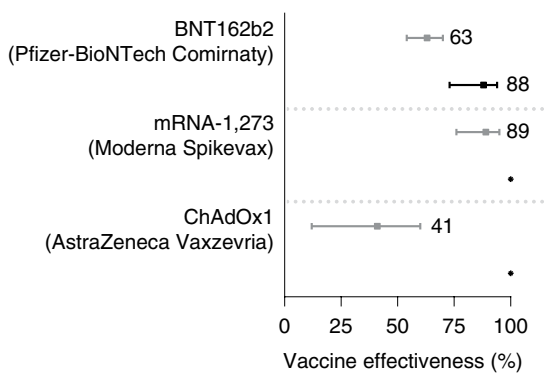

f

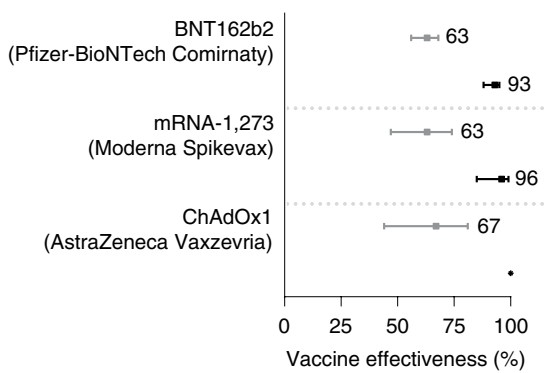

$\geq 14 \mathrm{~d}$ after dose 1

$\geq 7 \mathrm{~d}$ after dose 2

Fig. 1 | Vaccine effectiveness against symptomatic infection. a-f, Adjusted vaccine effectiveness estimates of BNT162b2 (Pfizer-BioNTech Comirnaty), mRNA-1273 (Moderna Spikevax) and ChAdOx1 (AstraZeneca Vaxzevria) vaccines $\geq 14 \mathrm{~d}$ after the first dose (for individuals who received only one dose) and $\geq 7 \mathrm{~d}$ after the second dose against symptomatic SARS-CoV-2 infection caused by Alpha (a), Beta (b), Gamma (c), Beta/Gamma (d), Delta (e) and non-VOC (f) SARS-CoV-2 between 14 December 2020 and 3 August 2021 in Ontario, Canada; $n=44,688$ test-positive Alpha, $n=378$ test-positive Beta, $n=1,969$ test-positive Gamma, $n=1,269$ test-positive Beta/Gamma, $n=3,136$ test-positive Delta and $n=31,440$ test-positive non-VOC SARS-CoV-2 symptomatic cases, and $n=599,191$ symptomatic test-negative controls. Data are presented as effectiveness point estimates, with error bars indicating the corresponding $95 \% \mathrm{Cls}$. Asterisks indicate vaccine effectiveness estimated as $100 \%$ on the basis of zero vaccinated test-positive cases.

\section{Results}

Study population. Over the study period, we identified 682,071 symptomatic community-dwelling individuals who were tested for SARS-CoV-2, with 31,440 (5\%) individuals positive for non-VOC SARS-CoV-2 and 51,440 (8\%) individuals positive for a VOC (Supplementary Table 1). Cases of Delta infections were younger, more likely to reside in the Central West region, more likely to occur later in the study period, less likely to have any comorbidities, and more likely to reside in neighbourhoods with lower household income and greater proportions of essential workers than cases of other VOC and non-VOC SARS-CoV-2 infections, as well as test-negative controls.

We identified 15,269 individuals with a COVID-19 hospitalization or death (Supplementary Table 2). We observed largely the same socio-demographic patterns between individuals with severe outcomes caused by Delta versus those caused by other VOC and non-VOC SARS-CoV-2.

Vaccine effectiveness against symptomatic infection. Vaccine effectiveness against symptomatic infection caused by Alpha, at $\geq 14 \mathrm{~d}$ after the first dose, was higher for mRNA-1273 (82\%; $95 \%$ CI, 80-84\%) than for BNT162b2 (67\%; 95\% CI, 65-68\%) and ChAdOx1 (63\%; 95\% CI, 59-66\%) (Fig. 1a). Vaccine effectiveness increased $\geq 7 \mathrm{~d}$ after the second dose against Alpha for all three vaccines: $\mathrm{mRNA}-1273=92 \%$ (95\% CI, $88-95 \%)$, BNT162b2 $=89 \%$ (95\% CI, 87-90\%) and ChAdOx1 =91\% (95\% CI, 62-98\%).

Estimates of effectiveness against symptomatic infection caused by Beta (Fig. 1b) were imprecise or were $100 \%$ because of low numbers or absence of vaccinated test-positive cases, respectively (Supplementary Table 3). Vaccine effectiveness against symptomatic infection caused by Gamma was higher after the first dose of mRNA-1273 (89\%; 95\% CI, 76-95\%) than after the first dose of ChAdOx1 (41\%; 95\% CI, 12-60\%), and was intermediate for BNT162b2 (63\%; 95\% CI, 54-70\%) (Fig. 1c). Effectiveness against Gamma increased after the second dose for BNT162b2 but could not be reliably estimated for mRNA-1273 and ChAdOx1 because of zero vaccinated cases. Protection against symptomatic infection caused by Beta/Gamma appeared to be marginally higher (but with overlapping confidence intervals) after the first dose of mRNA1273 (75\%; 95\% CI, 53-86\%) than after the first dose of BNT162b2 (64\%; 95\% CI, 54-72\%) and ChAdOx1 (62\%; 95\% CI, 37-77\%) (Fig. 1d). Receipt of the second dose increased vaccine effectiveness against Beta/Gamma for BNT162b2 (82\%; 95\% CI, 65-91\%) and mRNA-1273 (89\%; 95\% CI, 21-98\%) but could not be reliably estimated for ChAdOx1 due to absence of any vaccinated cases (Supplementary Table 3).

Against Delta, vaccine effectiveness after the first dose was higher for mRNA-1273 (70\%; 95\% CI, 64-76\%) and ChAdOx1 (68\%; 95\% CI, 57-76\%) than for BNT162b2 (57\%; 95\% CI, 53-61\%) (Fig. 1e). Vaccine effectiveness increased after the second dose for all three vaccines, to $95 \%$ (95\% CI, 91-97\%) for mRNA-1273, 87\% (95\% CI, 69-95\%) for ChAdOx1 and 92\% (95\% CI, 90-94\%) for BNT162b2.

By product, vaccine effectiveness after one dose tended to be lower against Delta than against Alpha and Gamma for mRNA$1273(70 \%$ vs $82 \%$ and $89 \%)$ and for BNT162b2 (57\% vs $67 \%$ and $63 \%)$, but was similar to effectiveness after one dose against Alpha for ChAdOx1 (68\% vs 63\%). Two doses of BNT162b2 and mRNA-1273 increased protection against Delta (92-95\%) to levels comparable to protection against Alpha (89-92\%), Beta (87\%) and Gamma (88\%). 

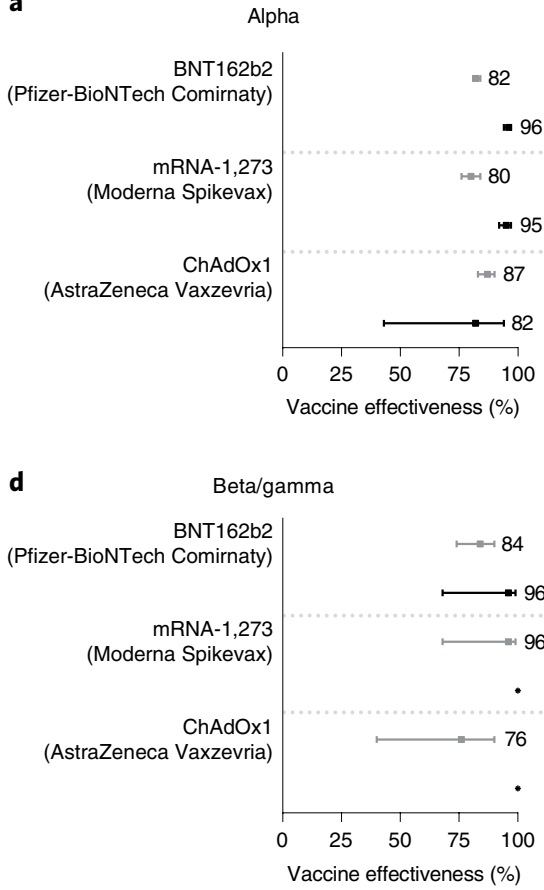

b

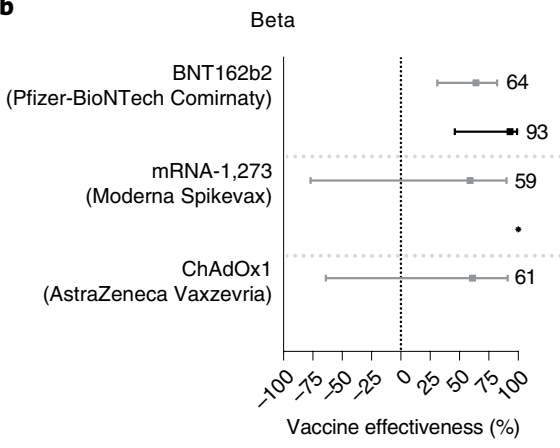

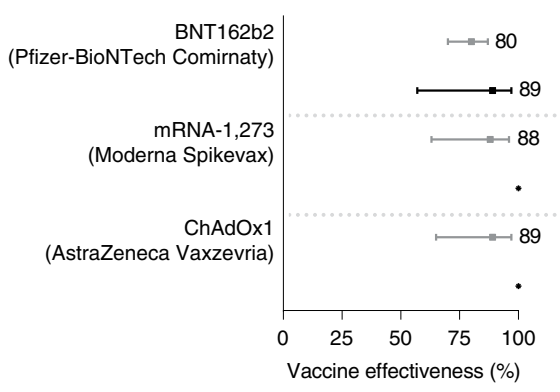

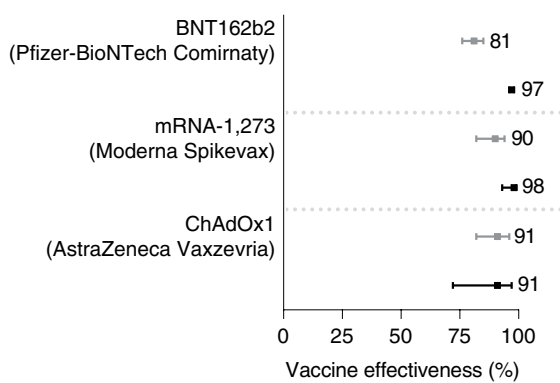

Non-VOC

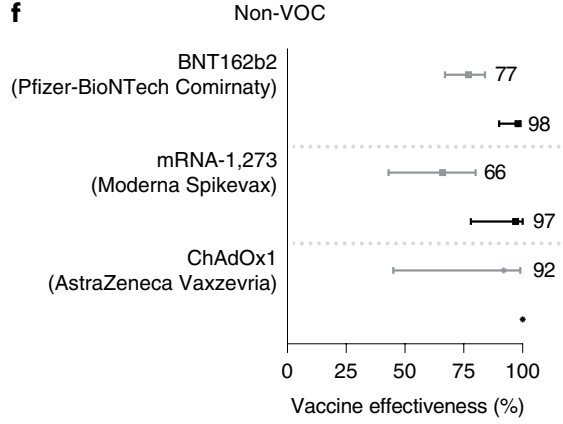

$\geq 14 \mathrm{~d}$ after dose 1

$\geq 7 \mathrm{~d}$ after dose 2

Fig. 2 | Vaccine effectiveness against severe outcomes (hospitalization or death). a-f, Adjusted vaccine effectiveness estimates of BNT162b2 (Pfizer-BioNTech Comirnaty), mRNA-1273 (Moderna Spikevax) and ChAdOx1 (AstraZeneca Vaxzevria) vaccines $\geq 14 \mathrm{~d}$ after the first dose (for individuals who received only one dose) and $\geq 7 \mathrm{~d}$ after the second dose against severe outcomes (hospitalization or death) caused by Alpha (a), Beta (b), Gamma (c), Beta/Gamma (d), Delta (e) and non-VOC (f) SARS-CoV-2 between 14 December 2020 and 17 August 2021 in Ontario, Canada; $n=7,438$ test-positive Alpha, $n=174$ test-positive Beta, $n=410$ test-positive Gamma, $n=237$ test-positive Beta/Gamma, $n=639$ test-positive Delta and $n=6,371$ test-positive non-VOC SARS-CoV-2 cases with severe outcomes (hospitalization or death), and $n=598,905$ symptomatic test-negative controls. Data are presented as effectiveness point estimates, with error bars indicating the corresponding $95 \% \mathrm{Cls}$. Asterisks indicate vaccine effectiveness estimated as $100 \%$ on the basis of zero vaccinated test-positive cases.

Vaccine effectiveness against hospitalization or death. Vaccine effectiveness against hospitalization or death caused by all four VOC was generally higher than against symptomatic infection after the first dose for all three vaccines (Fig. 2). In particular, against Delta, vaccine effectiveness against severe outcomes after the first dose of BNT162b2, mRNA-1273 and ChAdOx1 was 81\% (95\% CI, 76-85\%), 90\% (95\% CI, 82-94\%) and 91\% (95\% CI, 72-97\%), respectively (Fig. 2e). Receipt of the second dose was associated with vaccine effectiveness estimates above $90 \%$ against: Alpha, Beta and Delta for BNT162b2; Alpha and Delta for mRNA-1273; and Delta for ChAdOx1. Estimates were in the $80 \%$ range against Gamma for BNT162b2 and Alpha for ChAdOx1, and could not be reliably estimated for other VOC-vaccine combinations due to low numbers or absence of vaccinated cases.

Sensitivity analyses. In sensitivity analyses, vaccine effectiveness estimates using $\geq 21 \mathrm{~d}$ after the first dose and $\geq 14 \mathrm{~d}$ after the second dose were found to be mostly very similar (estimates differed by $\leq 5 \%$ ) to our primary analyses (Table 1 ). Where differences were $>5 \%$, the sensitivity analyses yielded higher estimates. Similar vaccine effectiveness estimates were also observed when limiting the study period to 5 April 2021 to 3 August 2021 (Supplementary Table 4).

Vaccine effectiveness by age group. In age-group-stratified analyses, vaccine effectiveness against symptomatic infection caused by all VOC was in most instances lower or similar in older adults (age $\geq 60$ years) compared with younger individuals (age $<60$ years) after partial vaccination (Supplementary Table 5). Vaccine effectiveness in older adults increased to levels comparable to those in younger individuals after the second dose, except against Gamma for BNT162b2 and against Delta for ChAdOx1. Vaccine effectiveness was higher against hospitalization and death than against symptomatic infection for both older and younger adults.

\section{Discussion}

We estimated that one dose of BNT162b2 and mRNA-1273 was $>50 \%$ and $>70 \%$ effective, respectively, against symptomatic infection caused by VOC that have circulated to date in Ontario, Canada. One dose of ChAdOxl prevented $41 \%$ of symptomatic infections by Gamma, and was $>60 \%$ effective against Alpha and Delta. For all three vaccines, effectiveness increased (to $>80 \%$ and, in most cases, $>90 \%$ ) following the second dose. Effectiveness of the first dose was substantially higher against hospitalization or death than against symptomatic infection for all VOC-vaccine combinations except for mRNA-1273 against Alpha and Gamma because vaccine effectiveness against symptomatic infection was already high; the second dose of mRNA vaccines further improved effectiveness against severe outcomes. We also found that: assessing effectiveness $\geq 21 \mathrm{~d}$ after the first dose and $\geq 14 \mathrm{~d}$ after the second dose resulted in similar or higher estimates, restricting the analysis to a period when all VOC were co-circulating yielded similar results, and the effectiveness of one dose tended to be lower for older adults than for younger adults.

Our vaccine effectiveness estimates against symptomatic COVID19 infection with Alpha and Gamma after the first dose of mRNA or ChAdOx1 vaccines are similar to estimates against infection from British Columbia, Canada (67\% for mRNA vaccines against Alpha and $61 \%$ against Gamma) ${ }^{15}$ and Qatar (88\% for mRNA-1273 against 
Table 1 | Vaccine effectiveness against Alpha (B.1.1.7), Beta (B.1.351), Gamma (P.1), Beta/Gamma and Delta (B.1.617.2) variants of concern by outcome, vaccine product, number of doses received, and time between most recent vaccination date and index date for those tested for SARS-CoV-2 between 14 December 2020 and 3 August 2021 in Ontario, Canada

Outcome Vaccine effectiveness ${ }^{\mathrm{a}}(95 \% \mathrm{Cl})$

\begin{tabular}{|c|c|c|c|c|c|c|}
\hline & Alpha & Beta & Gamma & Beta/Gamma & Delta $^{c}$ & Non-VOC SARS-CoV-2 \\
\hline \multicolumn{7}{|l|}{ Symptomatic infection } \\
\hline \multicolumn{7}{|c|}{ BNT162b2 (Pfizer-BioNTech Comirnaty) ${ }^{d}$} \\
\hline$\geq 21 \mathrm{~d}$ after one dose only & $70(69,72)$ & $48(6,71)$ & $67(57,74)$ & $68(57,76)$ & $59(54,63)$ & $65(58,71)$ \\
\hline$\geq 7 \mathrm{~d}$ after two doses & $89(87,90)$ & $87(8,98)$ & $88(73,94)$ & $82(65,91)$ & $92(90,94)$ & $93(88,95)$ \\
\hline$\geq 14 \mathrm{~d}$ after one dose only & $82(80,84)$ & $-^{\mathrm{e}}$ & $89(76,95)$ & $75(53,86)$ & $70(64,76)$ & $63(47,74)$ \\
\hline$\geq 21 \mathrm{~d}$ after one dose only & $83(80,85)$ & $-^{e}$ & $90(73,96)$ & $74(48,87)$ & $69(62,75)$ & $70(51,81)$ \\
\hline$\geq 7 \mathrm{~d}$ after two doses & $92(88,95)$ & $--^{f}$ & $--^{f}$ & $89(21,98)$ & $95(91,97)$ & $96(85,99)$ \\
\hline$\geq 14 \mathrm{~d}$ after two doses & $92(87,95)$ & $--^{f}$ & $--^{f}$ & $88(10,98)$ & $94(90,97)$ & $98(83,100)$ \\
\hline$\geq 14 \mathrm{~d}$ after two doses & $87(47,97)$ & $--^{f}$ & $--^{f}$ & $-^{e}$ & $88(68,96)$ & $-{ }^{f}$ \\
\hline \multicolumn{7}{|l|}{ Hospitalization or death } \\
\hline \multicolumn{7}{|c|}{ BNT162b2 (Pfizer-BioNTech Comirnaty) ${ }^{d}$} \\
\hline$\geq 14 \mathrm{~d}$ after one dose only & $82(81,84)$ & $64(31,82)$ & $80(70,87)$ & $84(74,90)$ & $81(76,85)$ & $77(67,84)$ \\
\hline$\geq 21 \mathrm{~d}$ after one dose only & $87(85,88)$ & $65(23,84)$ & $88(79,93)$ & $87(77,92)$ & $81(76,85)$ & $88(79,94)$ \\
\hline$\geq 7 \mathrm{~d}$ after two doses & $96(94,97)$ & $93(46,99)$ & $89(57,97)$ & $96(68,99)$ & $97(96,98)$ & $98(90,99)$ \\
\hline$\geq 14 \mathrm{~d}$ after two doses & $96(94,97)$ & $92(39,99)$ & $94(59,99)$ & $95(64,99)$ & $98(96,99)$ & $97(88,99)$ \\
\hline \multicolumn{7}{|c|}{ mRNA-1273 (Moderna Spikevax) } \\
\hline$\geq 14 \mathrm{~d}$ after one dose only & $80(76,84)$ & $59(-77,90)$ & $88(63,96)$ & $96(68,99)$ & $90(82,94)$ & $66(43,80)$ \\
\hline$\geq 7 \mathrm{~d}$ after two doses & $82(43,94)$ & $-{ }^{\mathrm{e}}$ & $--^{f}$ & $--^{f}$ & $91(72,97)$ & $--^{f}+$ \\
\hline$\geq 14 \mathrm{~d}$ after two doses & $92(41,99)$ & $-\mathrm{e}$ & $-f$ & $--^{f}$ & $90(67,97)$ & $-f$ \\
\hline
\end{tabular}

adjusted for age, sex, public health unit region, period of test (weekly period for Delta, and biweekly period for non-VOC SARS-CoV-2 and other VOC), number of SARS-CoV-2 tests in the 3 months before 14 December 2020, presence of any comorbidity that increase the risk of severe COVID-19, receipt of 2019/2020 and/or 2020/2021 influenza vaccination, and Census dissemination area-level quintiles

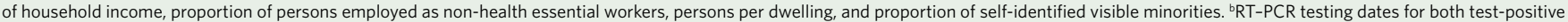
cases and test-negative controls were restricted to 11 January 2021 to 3 August 2021 for Beta, Gamma, and Beta/Gamma. CRT-PCR testing dates for both test-positive cases and test-negative controls were restricted to 5 April 2021 to 3 August 2021 for Delta. ${ }^{~}$ For one dose only, excludes individuals who received two doses; shorter intervals after the doses include the longer interval (that is, analyses for $\geq 14 \mathrm{~d}$ after the first dose also includes subjects who were $\geq 21 \mathrm{~d}$ after the first dose, and analyses for $\geq 7 \mathrm{~d}$ also includes subjects who were $\geq 14 \mathrm{~d}$ after the second dose). eVaccine effectiveness not reported due to extremely imprecise $95 \%$ confidence intervals. ${ }^{f}$ Vaccine effectiveness estimated as $100 \%$ on the basis of zero vaccinated test-positive cases.

Alpha $)^{9}$ but higher than estimates after one dose against infection or symptomatic COVID-19 reported from Qatar (for BNT162b2, 30\% against Alpha and 17\% against Beta $; 61 \%$ for mRNA-1273 against Beta $^{9}$ ), England (49\% for BNT162b2 and 51\% for ChAdOx1 against Alpha $)^{10}$ and Scotland (27\% for BNT162b2 and 39\% for ChAdOx1 against Alpha) ${ }^{6}$. Our estimates after one dose are also higher than the estimates for BNT162b2 against severe, critical, or fatal disease in Qatar (54\%) ${ }^{7}$, but comparable with the estimates for BNT162b2 or ChAdOx1 against hospitalization caused by the Alpha variant in England $(83 \%)^{8}$ and for mRNA-1273 against severe, critical, or fatal disease caused by Alpha/Beta in Qatar (82\%) ${ }^{9}$.
We estimated higher vaccine effectiveness against symptomatic COVID-19 infection with the Delta variant after one dose of mRNA or ChAdOx1 vaccines than the effectiveness against symptomatic or asymptomatic infections reported from England and Scotland (30$33 \%$ for BNT162b2 and $18-33 \%$ for ChAdOx1 $)^{6,10}$. However, our effectiveness estimates against symptomatic infections with Delta after the first dose were lower than the estimates against infections in Qatar (64\% for BNT162b2 and 79\% for mRNA-1273) ${ }^{11}$. Our estimates after the second dose were higher than the estimates against symptomatic infections reported in England (88\% for BNT162b2 and $67 \%$ for ChAdOx1) ${ }^{10}$, Scotland (83\% for BNT162b2 and $61 \%$ 
for ChAdOx1) ${ }^{6}$, Israel $\left(40.5 \%\right.$ for BNT162b2) ${ }^{16}$, the USA (42\% for BNT162b2 and 76\% for mRNA-1273 against infection $)^{17}$ and Qatar (53.5\% for BNT162b2 and $84.8 \%$ for mRNA-1273 $)^{11}$. Against hospitalization with the Delta variant, our vaccine effectiveness after one dose was lower for BNT162b2 and higher for ChAdOx1 than the estimates from England (81\% vs $94 \%$ for BNT162b2 and $91 \%$ vs $71 \%$ for $\mathrm{ChAdOx} 1)^{8}$. Vaccine effectiveness against severe, critical and fatal COVID-19 disease with Delta after one dose of BNT162b2 and mRNA-1273 in Qatar was reported to be $100 \%$ because of zero vaccinated cases ${ }^{11}$.

After the second dose, our estimates against both outcomes for all VOC were higher than after one dose, and comparable with estimates reported in previous studies ${ }^{6-8,10}$. However, vaccine effectiveness after two doses was reported to be lower than after one dose against any infection with Delta (54\% vs $64 \%$ ) in Qatar, which the authors interpreted as waning of protection ${ }^{11}$.

The heterogeneity in vaccine estimates, particularly after one dose, across studies could result from a number of factors, including differences in study design, study population, SARS-CoV-2 test assays and testing criteria, comprehensiveness of test results recorded in databases, outcome definitions and ascertainment, timing of VOC circulation, vaccine priority groups, vaccine roll-out, interval between vaccine doses, and variables adjusted to control for possible confounding ${ }^{18}$.

Province-wide data allowed us to estimate the effectiveness of all three vaccines used in Canada against symptomatic infection and severe outcomes caused by the four VOC that have circulated in Canada thus far. The test-negative study design has the advantage of controlling for bias resulting from differences in healthcare-seeking behaviour between vaccinated and unvaccinated individuals ${ }^{19}$.

There are some limitations of our study. First, VOC classification in this study relied on a combination of mutation screening and whole-genome sequencing, and the criteria for sequencing evolved over time. Our definition of Delta specimens initially relied largely on a proxy measure of an N501Y-/E484K- result on mutation screening and a combination of date and geographic location, which were used to infer probable Delta variant specimens. Thus, a small proportion of specimens classified as Delta may have been non-VOC SARS-CoV-2 specimens. Second, since vaccine effectiveness is probably impacted by age, interval between vaccine doses, time between vaccine receipt and index date, vaccine product, and VOC, and given that the eligibility criteria for vaccination (for example, initial prioritization of older age groups), the availability of certain vaccine products, and the distribution of circulating VOC all varied over time, comparisons of vaccine effectiveness estimates between combinations of vaccine products and VOC should be made with caution. However, we included a sensitivity analysis that restricted the study period to individuals tested during 5 April through 3 August 2021 (to ensure that all VOC and non-VOC SARS-CoV-2 were circulating and to mitigate temporal confounding caused by the aforementioned factors), which yielded very similar results to our primary analysis. Third, it is possible that we may have under-ascertained severe outcomes if they were not recorded in the public health surveillance database used, such as when severe outcomes occurred after completion of case follow-up or when case volumes exceeded public health system capacity and public health investigation of each laboratory-confirmed case was not possible. This may have resulted in overestimation of vaccine effectiveness against severe outcomes. Fourth, we used specimen collection date as the index date because of lack of available data on symptom onset date in the Ontario Laboratories Information System (OLIS), which precluded us from restricting the study population to individuals who were tested within $10 \mathrm{~d}$ of symptom onset. Thus, we may have underestimated vaccine effectiveness by increasing the risk of false-negative cases by extending the interval between symptom onset and testing. Lastly, despite our best efforts to adjust for potential confounders and the use of the test-negative design, these results may nonetheless be susceptible to residual confounding, given the observational nature of the study.

In conclusion, our real-world vaccine effectiveness estimates suggest that even a single dose of these three COVID-19 vaccine products provides considerable protection against symptomatic infection and severe outcomes caused by these four VOC, particularly for young adults, and that two doses provide even higher protection. While vaccine effectiveness estimates after two doses are at present most relevant to high-income countries with adequate vaccine supply and relatively higher two-dose vaccine coverage, effectiveness estimates after one dose remain relevant for low- and middle-income countries with suboptimal vaccine supply and low one- or two-dose vaccine coverage. Thus, our findings have public health policy implications worldwide. Jurisdictions facing COVID19 vaccine supply constraints may benefit from delaying the second dose (at least for younger individuals) to maximize the number of individuals receiving partial protection from the first dose, thereby potentially providing greater overall protection of the population more rapidly. However, older adults would likely benefit most from minimizing the delay in receiving the second dose to achieve adequate protection against VOC, including Delta, the predominant VOC currently in circulation worldwide.

\section{Methods}

We employed a test-negative design to compare vaccination status between test-positive individuals (with symptomatic infection or a severe outcome) and symptomatic but test-negative individuals ${ }^{19}$. We included community-dwelling Ontarians aged $\geq 16$ years who had symptoms consistent with, or a severe outcome attributable to, COVID-19, and who were tested for SARS-CoV-2 between 14 December 2020 and 3 August 2021. We excluded individuals who had tested positive for SARS-CoV-2 before their selected index date and those who received Ad26.COV2.S (Janssen), because it has not been used in Ontario (despite receiving approval), or mixed (ChAdOx1-mRNA or different mRNA) vaccine schedules.

Data sources and definitions. Comprehensive province-wide datasets for SARS-CoV-2 laboratory testing, SARS-CoV-2 public health surveillance, COVID-19 vaccination and healthcare system use were linked using unique encoded identifiers and analysed at ICES (formerly the Institute for Clinical Evaluative Sciences). Details have been described previously ${ }^{20}$.

Vaccination status. We obtained information regarding COVID-19 vaccination status, including vaccine product, date of administration and dose number from COVaxON, a centralized COVID-19 vaccine information system in Ontario.

COVID-19 testing and identification of variants. Data on laboratory-confirmed SARS-CoV-2 infection detected by real-time reverse transcription polymerase chain reaction (RT-PCR) were collected from OLIS for both individuals who tested positive (treated as cases) and individuals who tested negative (treated as controls). We used specimen collection date as the index date because symptom onset date was not consistently available in OLIS. We used the first positive test for cases and a randomly selected negative test for controls, with multiple negative tests during the study period.

We obtained information on variants from the Public Health Case and Contact Management system (CCM), which contains results of screening tests for mutations and whole-genome sequencing to assign SARS-CoV-2 lineage or VOC. All RT-PCR positive specimens with cycle threshold values $\leq 35$ were screened for $\mathrm{N} 501 \mathrm{Y}$ and E484K mutations by multiplex RT-PCR (VOC PCR) ${ }^{21}$. From 7 June 2021, specimens positive for both N501Y and E484K mutations were also screened for K417N and K417T mutations to differentiate specimens between Beta and Gamma variants ${ }^{21}$

At the beginning of 2021, whole-genome sequencing was performed on specimens that had specific mutations detected by VOC PCR to confirm that they were indeed VOC. From 3 February 2021, specimens with the N501Y mutation, and from 22 March 2021, all specimens with the E484K mutation and $5 \%$ of E484K-negative specimens (all with cycle threshold values $\leq 30$ ) were sequenced for surveillance purposes ${ }^{21,22}$. A subset of RT-PCR-positive specimens without any mutations detected by VOC PCR was also selected for sequencing for surveillance purposes ${ }^{21}$. Additionally, VOC PCR testing and sequencing were performed for specific indications, such as recent travellers, partially or fully vaccinated individuals, cases of suspected re-infection, or to support investigations of outbreaks and potential super-spreading events ${ }^{23}$. Ontario started sequencing $10 \%$ and $50 \%$ of VOC PCR-screened specimens on 2 May 2021 and 30 May 2021, respectively. From 14 June 2021, Ontario started sequencing $100 \%$ of eligible VOC 
PCR-screened specimens ${ }^{21}$, which continued until the end of the study period before scaling back to sequencing $50 \%$ of specimens on 27 August $2021^{22}$.

In addition to those classified into SARS-CoV-2 lineages based on sequencing, we considered specimens positive for the N501Y mutation and negative for the E484K mutation (N501Y+/E484K-) as Alpha. Both Beta and Gamma have N501Y and $\mathrm{E} 484 \mathrm{~K}$ mutations, and can only be differentiated by additional screening for K417N and K417T mutations. Hence, we considered specimens positive for N501Y, E484K and K417N mutations as Beta, and specimens positive for N501Y, E484K and K417T as Gamma. We grouped the specimens that could not be separated into Beta and Gamma into a combined Beta/Gamma group. We classified specimens collected after 1 April 2021 that were negative for both N501Y and E484K (N501Y-/E484K-) mutations as either probable, possible, or unlikely Delta cases based on the predicted probability that it was Delta. To do this, we created a logistic regression model of the probability that an N501Y-/E484K- case was Delta based on the date of specimen collection and the forward sortation area (geographical unit based on the first three characters of the postal code) ranked by the cumulative incidence of laboratory-confirmed SARS-CoV-2 cases between 23 January 2020 and 28 March 2021 and grouped into deciles ${ }^{24}$. For each decile, we examined the trajectories of the daily counts of N501Y-/E484K- specimens between 1 April 2021 and 30 May 2021 to estimate the predicted probability that an N501Y-/E484K- specimen represented Delta. We classified specimens with $>75 \%$ probability of being a Delta case to be 'probable Delta' cases, those with $25-75 \%$ probability to be 'possible Delta' cases, and those with $<25 \%$ probability to be 'unlikely Delta' cases. Our approach correlates well with sequencing results $(n=538)$ for the province, indicating a rapid increase in the proportion of N501Y-/E484K- cases being identified as Delta from mid-March to mid-May $2021^{25}$. As of 31 May 2021, all N501Y-/E484K- specimens were considered Delta $^{22}$. We grouped the probable Delta cases with those identified through sequencing. We classified specimens with no lineage information and N501Y-I E484K- specimens collected before 1 April 2021 as non-VOC SARS-CoV-2. We also grouped the 'unlikely Delta' cases with the non-VOC specimens. We excluded 'possible Delta' cases and N501Y-/E484K+ cases from our analyses.

Outcomes. For vaccine effectiveness against symptomatic infection, individuals who were symptomatic and tested positive for SARS-CoV-2 in OLIS were considered as cases. For severe outcomes, test-positive individuals who had a hospitalization or death up to 17 August 2021 (regardless of the presence of any symptoms recorded at the time of RT-PCR testing) were identified from CCM and considered as cases. Individuals who were symptomatic but only had tests negative for SARS-CoV-2 in OLIS were considered as controls for both outcomes. However, for severe outcomes, we excluded symptomatic test-negative individuals who later tested positive between 4 and 17 August 2021.

Covariates. We obtained information on the following covariates from administrative databases: age and sex from the Ontario Registered Persons Database (RPDB); postal code and Public Health Unit of residence from the RPDB and Statistics Canada Postal Code Conversion File Plus (version 7B); the number of SARS-CoV-2 RT-PCR tests for each individual during the 3 months before 14 December (a proxy for individuals who are at increased risk of exposure to SARS-CoV-2 infection and undergo frequent testing), and biweekly (weekly for Delta) period of RT-PCR test to account for the temporal viral activity and regional vaccine roll-out, created using testing information from OLIS; comorbidities ${ }^{26}$ associated with increased risk of severe COVID-19, identified from various databases using validated algorithms and commonly used diagnostic codes and algorithms described previously ${ }^{27}$, including Expanded Diagnostic Clusters and Special Population Markers from the Johns Hopkins ACG System (version 10) ${ }^{28}$; influenza vaccination status during the 2019/2020 and/or 2020/2021 influenza season (a proxy for health behaviours), determined from physician and pharmacist billing claims in the Ontario Health Insurance Plan and Ontario Drug Benefit databases, respectively; and neighbourhood-level information on median household income, proportion of the working population employed as non-health essential workers, average number of persons per dwelling and proportion of the population who self-identify as a visible minority, obtained from 2016 Census data. Details regarding these covariates are provided in Supplementary Table 6 (ref. ${ }^{20}$ ).

Statistical analyses. We used multivariable logistic regression models to estimate the odds ratio comparing the odds of vaccination in test-positive cases with the odds of vaccination among test-negative controls, adjusting for the aforementioned covariates that are associated with COVID-19 and vaccine uptake ${ }^{26,29,30}$. We calculated vaccine effectiveness using the following formula: Vaccine effectiveness $=1-($ odds ratio $) \times 100 \%$.

We estimated vaccine effectiveness against SARS-CoV-2 infection and severe COVID-19 outcomes (hospitalization or death) caused by non-VOC SARS-CoV-2, Alpha, Beta, Gamma, Beta/Gamma and Delta separately by vaccine product (BNT162b2, mRNA-1273 and ChAdOx1) and number of doses received. For individuals who had received only one dose by the index date, we calculated vaccine effectiveness $\geq 14 \mathrm{~d}$ after the first dose. For individuals who had received two doses, we calculated vaccine effectiveness $\geq 7 \mathrm{~d}$ after the second dose. As a sensitivity analysis, and to facilitate comparisons with other studies, we also estimated vaccine effectiveness $\geq 21 \mathrm{~d}$ after the first dose and $\geq 14 \mathrm{~d}$ after the second dose.

When estimating vaccine effectiveness against Beta, Gamma, Beta/Gamma and Delta, we restricted both test-positive cases and test-negative controls to those who were tested on/after the dates of initial confirmation of these variants in Ontario (11 January 2021 for Beta, Gamma and Beta/Gamma; 5 April 2021 for Delta). Furthermore, since the primary periods of circulation for non-VOC SARS-CoV-2 and VOC varied relative to the vaccination campaign (that is, more non-VOC SARS-CoV-2 circulated earlier in the campaign when fewer individuals were vaccinated, whereas Delta circulated later when more were vaccinated), we conducted a sensitivity analysis restricted to individuals who were tested between 5 April 2021 and 3 August 2021 when non-VOC SARS-CoV-2 and VOC were concurrently circulating, thereby accounting for differences in vaccine availability and coverage over time.

Lastly, we estimated vaccine effectiveness stratified by age group ( $<60$ years and $\geq 60$ years).

All analyses were conducted using SAS version 9.4 (SAS Institute). All tests were two-sided and used $P<0.05$ as the level of statistical significance. We did not report estimates of vaccine effectiveness when $95 \%$ confidence intervals (CIs) were extremely imprecise (that is, ranging between a very large negative number and nearly 100) or when vaccine effectiveness was estimated as $100 \%$ on the basis of zero vaccinated test-positive cases and the $95 \%$ CIs were essentially infinite.

Ethics approval. ICES is a prescribed entity under Ontario's Personal Health Information Protection Act (PHIPA). Section 45 of PHIPA authorizes ICES to collect personal health information, without consent, for the purpose of analysis or compiling statistical information with respect to the management, evaluation or monitoring of the allocation of resources to or planning for all or part of the health system. Projects that use data collected by ICES under section 45 of PHIPA, and use no other data, are exempt from Research Ethics Board review. The use of the data in this project is authorized under section 45 and approved by ICES' Privacy and Legal Office.

Reporting Summary. Further information on research design is available in the Nature Research Reporting Summary linked to this article.

\section{Data availability}

The dataset from this study is held securely in coded form at ICES. While legal data sharing agreements between ICES and data providers (for example, healthcare organizations and government) prohibit ICES from making the dataset publicly available, access may be granted to those who meet pre-specified criteria for confidential access, available at www.ices.on.ca/DAS (email: das@ices.on.ca). Source data are provided with this paper.

\section{Code availability}

The full dataset creation plan and underlying analytic code are available from the authors upon request, understanding that the computer programmes may rely upon coding templates or macros that are unique to ICES and are therefore either inaccessible or may require modification.

Received: 6 August 2021; Accepted: 16 December 2021; Published online: 7 February 2022

\section{References}

1. Weekly Epidemiological Update on COVID-19 2021 edn 43 (WHO, accessed 18 June 12021); https://apps.who.int/iris/bitstream/handle/10665/341716/ CoV-weekly-sitrep8Jun21-eng.pdf.pdf?sequence $=1$

2. Weekly Epidemiological Update on COVID-19 2021 edn 59 (WHO, accessed 29 September 2021); https://www.who.int/publications/m/item/ weekly-epidemiological-update-on-covid-19-28-september-2021

3. Emary, K. R. W. et al. Efficacy of ChAdOx1 nCoV-19 (AZD1222) vaccine against SARS-CoV-2 variant of concern 202012/01 (B.1.1.7): an exploratory analysis of a randomised controlled trial. Lancet 397, 1351-1362 (2021).

4. Madhi, S. A. et al. Efficacy of the ChAdOx1 nCoV-19 Covid-19 vaccine against the B.1.351 Variant. N. Engl. J. Med. 384, 1885-1898 (2021).

5. Thomas, S. J. et al. Safety and efficacy of the BNT162b2 mRNA Covid-19 vaccine through 6 months. N. Engl. J. Med. https://doi.org/10.1056/ NEJMoa2110345 (2021).

6. Sheikh, A. et al. SARS-CoV-2 Delta VOC in Scotland: demographics, risk of hospital admission, and vaccine effectiveness. Lancet 397, 2461-2462 (2021).

7. Abu-Raddad, L. J., Chemaitelly, H. \& Butt, A. A. Effectiveness of the BNT162b2 Covid-19 vaccine against the B.1.1.7 and B.1.351 Variants. N. Engl. J. Med. https://doi.org/10.1056/NEJMc2104974 (2021).

8. Stowe, J. et al. Effectiveness of COVID-19 vaccines against hospital admission with the Delta (B.1.617.2) variant. Preprint at https://media.tghn.org/articles/ Effectiveness_of_COVID-19_vaccines_against_hospital_admission_with_the Delta_B._G6gnnqJ.pdf (2021). 
9. Chemaitelly, $\mathrm{H}$. et al. mRNA-1273 COVID-19 vaccine effectiveness against the B.1.1.7 and B.1.351 variants and severe COVID-19 disease in Qatar. Nat. Med. https://doi.org/10.1038/s41591-021-01446-y (2021).

10. Lopez Bernal, J. et al. Effectiveness of Covid-19 vaccines against the B.1.617.2 (Delta) Variant. N. Engl. J. Med. 385, 585-594 (2021).

11. Tang, P. et al. BNT162b2 and mRNA-1273 COVID-19 vaccine effectiveness against the Delta (B.1.617.2) variant in Qatar. Nat. Med. 27, 2136-2143 (2021).

12. Ontario's COVID-19 Vaccination Plan (Ontario Ministry of Health, Accessed 14 June 2021); https://covid-19.ontario.ca/ontarios-covid-19vaccination-plan

13. Ontario Proceeding with Second Dose Administration of AstraZeneca (Queen's Printer for Ontario 2012-2021, Accessed 23 May 2021); https://news.ontario. $\mathrm{ca} / \mathrm{en} / \mathrm{release} / 1000174 /$ ontario-proceeding-with-second-dose-administrationof-astrazeneca\#quickfacts

14. Epidemiologic Summary: COVID-19 in Ontario - January 15, 2020 to September 22, 2021 Toronto, ON (Ontario Agency for Health Protection and Promotion (Public Health Ontario), Accessed 23 September 2021); https:// www.publichealthontario.ca/-/media/documents/ncov/epi/covid-19-dailyepi-summary-report.pdf?la $=$ en

15. Skowronski, D. M. et al. Single-dose mRNA vaccine effectiveness against SARS-CoV-2, including Alpha and Gamma variants: a test-negative design in adults 70 years and older in British Columbia, Canada. Clin. Infect. Dis. https://doi.org/10.1093/cid/ciab616 (2021).

16. COVID-19 Vaccine Effectiveness Against the Delta Variant Israel's Ministry of Health Report 2021 (Israel's Ministry of Health, Accessed 21 August 2021); https://www.gov.il/BlobFolder/reports/vaccine-efficacy-safety-follow-upcommittee/he/files publications corona two-dose-vaccination-data.pdf

17. Puranik, A. et al. Comparison of two highly-effective mRNA vaccines for COVID-19 during periods of Alpha and Delta variant prevalence. Preprint at medRxiv https://doi.org/10.1101/2021.08.06.21261707 (2021).

18. Evaluation of COVID-19 Vaccine Effectiveness: Interim Guidance, 17 March 2021 (WHO, accessed 28 March 2021); https://apps.who.int/iris/rest/ bitstreams/1337417/retrieve

19. Jackson, M. L. \& Nelson, J. C. The test-negative design for estimating influenza vaccine effectiveness. Vaccine 31, 2165-2168 (2013).

20. Chung, H. et al. Effectiveness of BNT162b2 and mRNA-1273 Covid-19 vaccines against symptomatic SARS-CoV-2 infection and severe Covid-19 outcomes in Ontario, Canada: test negative design study. Brit. Med. J. 374, n1943 (2021).

21. SARS-CoV-2 (COVID-19 Virus) Variant of Concern (VoC) Surveillance, Toronto, ON (Ontario Agency for Health Protection and Promotion (Public Health Ontario), accessed 25 March 2021); https://www.publichealthontario. $\mathrm{ca} / \mathrm{en} /$ laboratory-services/test-information-index/covid-19-voc

22. Epidemiologic Summary: SARS-CoV-2 Whole-Genome Sequencing in Ontario, September 21, 2021 Toronto, ON (Ontario Agency for Health Protection and Promotion (Public Health Ontario), accessed 27 September 2021); https://www.publichealthontario.ca/-/media/documents/ncov/epi/ covid-19-sars-cov2-whole-genome-sequencing-epi-summary.pdf? sc_lang=en

23. SARS-CoV-2 Variant of Concern Testing/Whole-Genome Sequencing Public Health Ontario Laboratory Information Form 2021 (Ontario Agency for Health Protection and Promotion (Public Health Ontario), Accessed 21 June 2021); https://www.publichealthontario.ca/-/media/documents/lab/ sars-cov-2-voc-screening-request-form.pdf?la=en

24. Brown, K. A. et al. A Strategy for the Mass Distribution of COVID19 Vaccines in Ontario Based on Age and Neighbourhood (Ontario COVID-19 Science Advisory Table, 2021); https://doi.org/10.47326/ocsat.2021.02.10.1.0

25. Brown, K. A. et al. Inflection in prevalence of SARS-CoV-2 infections missing the N501Y mutation as a marker of rapid Delta (B.1.617.2) lineage expansion in Ontario, Canada. Preprint at medRxiv https://doi.org/10.1101/2021.06.22. 21259349 (2021)

26. Underlying Medical Conditions Associated with High Risk for Severe COVID-19: Information for Healthcare Providers 2021 (Centers for Disease Control and Prevention (CDC), Accessed 12 April 2021); https://www.cdc. gov/coronavirus/2019-ncov/hcp/clinical-care/underlyingconditions.html
27. Kwong, J. C. et al. Can routinely collected laboratory and health administrative data be used to assess influenza vaccine effectiveness? Assessing the validity ofthe flu and other respiratory viruses research (FOREVER) cohort. Vaccine 37, 4392-4400 (2019).

28. The Johns Hopkins ACG System (Johns Hopkins Univ., Accessed 14 July 2021); http://www.hopkinsacg.org/

29. Guidance on the Prioritization of Key Populations for COVID-19 Immunization 2021 (National Advisory Committee on Immunization (NACI), Accessed May 3 2021); https://www.canada.ca/en/public-health/services/immunization/ national-advisory-committee-on-immunization-naci/guidance-prioritizationkey-populations-covid-19-vaccination.html

30. Sundaram, M. E. et al. Individual and social determinants of SARS-CoV-2 testing and positivity in Ontario, Canada: a population-wide study. CMAJ 193, E723-E734 (2021).

\section{Acknowledgements}

This work was supported by the Canadian Immunization Research Network (CIRN) through a grant from the Public Health Agency of Canada and the Canadian Institutes of Health Research (CNF 151944); funding from the Public Health Agency of Canada through the Vaccine Surveillance Reference Group and the COVID-19 Immunity Task Force; ICES, which is funded by an annual grant from the Ontario Ministry of Health $(\mathrm{MOH})$ and the Ministry of Long-Term Care (MLTC); and the Ontario Health Data Platform (OHDP), a Province of Ontario initiative to support Ontario's ongoing response to COVID-19 and its related impacts. J.C.K. was supported by a Clinician-Scientist Award from the University of Toronto Department of Family and Community Medicine. P.C.A. was supported by a Mid-Career Investigator Award from the Heart and Stroke Foundation. The study sponsors did not participate in the design and conduct of the study; collection, management, analysis and interpretation of the data; preparation, review or approval of the manuscript; or the decision to submit the manuscript for publication. Parts of this material are based on data and/or information compiled and provided by the Canadian Institute for Health Information (CIHI) and by Cancer Care Ontario (CCO). However, the analyses, conclusions, opinions and statements expressed herein are solely those of the authors and do not reflect those of the funding or data sources; no endorsement by ICES, MOH, MLTC, OHDP, its partners, the Province of Ontario, CIHI or CCO is intended or should be inferred. We thank Public Health Ontario for access to case-level data from CCM and COVID-19 laboratory data, as well as assistance with data interpretation; the staff of Ontario's public health units who are responsible for COVID-19 case and contact management and data collection within CCM; IQVIA Solutions Canada Inc. for use of their Drug Information Database; and the Ontario residents without whom this research would be impossible.

\section{Author contributions}

J.C.K. and H.C. designed and oversaw the study. S.N., S.H. and H.C. obtained the data and conducted all analyses (dataset and variable creation, and statistical modelling). B.C. contributed to data analyses and data preparation for the symptomatic dataset. H.C. and S.H. verified the data in the study. S.N. did the literature search. S.N. and J.C.K. drafted the manuscript. All authors contributed to the analysis plan, interpreted the results, critically reviewed and edited the manuscript, approved the final version and agreed to be accountable for all aspects of the work.

\section{Competing interests}

K.W. is CEO of CANImmunize and serves on the data safety board for the Medicago COVID-19 vaccine trial. The other authors declare no competing interests.

\section{Additional information}

Supplementary information The online version contains supplementary material available at https://doi.org/10.1038/s41564-021-01053-0.

Correspondence and requests for materials should be addressed to Jeffrey $\mathrm{C}$. Kwong.

Peer review information Nature Microbiology thanks the anonymous reviewers for their contribution to the peer review of this work.

Reprints and permissions information is available at www.nature.com/reprints.

Publisher's note Springer Nature remains neutral with regard to jurisdictional claims in published maps and institutional affiliations.

(c) The Author(s), under exclusive licence to Springer Nature Limited 2022

\title{
on behalf of the Canadian Immunization Research Network (CIRN) Provincial Collaborative Network (PCN) Investigators
}

\author{
Sharifa Nasreen ${ }^{1,2}$, Sarah A. Buchan ${ }^{1,2,3,4}$, Deshayne B. Fell ${ }^{1,5,6}$, Maria E. Sundaram ${ }^{1,2,8}$, \\ Kumanan Wilson ${ }^{10,11}$, Sarah E. Wilson ${ }^{1,2,3,4}$ and Jeffrey C. Kwong 1,2,3,12,13
}




\section{nature portfolio}

Corresponding author(s): Jeffrey C. Kwong

Last updated by author(s): Dec 12, 2021

\section{Reporting Summary}

Nature Portfolio wishes to improve the reproducibility of the work that we publish. This form provides structure for consistency and transparency in reporting. For further information on Nature Portfolio policies, see our Editorial Policies and the Editorial Policy Checklist.

\section{Statistics}

For all statistical analyses, confirm that the following items are present in the figure legend, table legend, main text, or Methods section.

n/a Confirmed

$\bigotimes$ The exact sample size $(n)$ for each experimental group/condition, given as a discrete number and unit of measurement

$\bigotimes$ A statement on whether measurements were taken from distinct samples or whether the same sample was measured repeatedly

The statistical test(s) used AND whether they are one- or two-sided

Only common tests should be described solely by name; describe more complex techniques in the Methods section.

$\bigotimes$ A description of all covariates tested

Х $\square$ A description of any assumptions or corrections, such as tests of normality and adjustment for multiple comparisons

$\checkmark$ A full description of the statistical parameters including central tendency (e.g. means) or other basic estimates (e.g. regression coefficient)

AND variation (e.g. standard deviation) or associated estimates of uncertainty (e.g. confidence intervals)

$\triangle$ For null hypothesis testing, the test statistic (e.g. $F, t, r$ ) with confidence intervals, effect sizes, degrees of freedom and $P$ value noted Give $P$ values as exact values whenever suitable.

Х $\square$ For Bayesian analysis, information on the choice of priors and Markov chain Monte Carlo settings

Х $\square$ For hierarchical and complex designs, identification of the appropriate level for tests and full reporting of outcomes

$\square$ Estimates of effect sizes (e.g. Cohen's $d$, Pearson's $r$ ), indicating how they were calculated

Our web collection on statistics for biologists contains articles on many of the points above.

\section{Software and code}

Policy information about availability of computer code

Data collection No software was used for data collection

Data analysis All analyses were conducted using SAS Version 9.4 (SAS Institute Inc., Cary, NC) at ICES. The full dataset creation plan and underlying analytic code are available from the authors upon request, understanding that the computer programs may rely upon coding templates or macros that are unique to ICES and are therefore either inaccessible or may require modification.

For manuscripts utilizing custom algorithms or software that are central to the research but not yet described in published literature, software must be made available to editors and

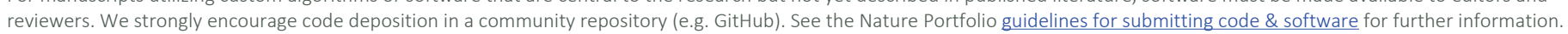

\section{Data}

Policy information about availability of data

All manuscripts must include a data availability statement. This statement should provide the following information, where applicable:

- Accession codes, unique identifiers, or web links for publicly available datasets

- A description of any restrictions on data availability

- For clinical datasets or third party data, please ensure that the statement adheres to our policy

The dataset from this study is held securely in coded form at ICES. While legal data sharing agreements between ICES and data providers (e.g., healthcare organizations and government) prohibit ICES from making the dataset publicly available, access may be granted to those who meet pre-specified criteria for confidential access, available at www.ices.on.ca/DAS (email: das@ices.on.ca). 
Please select the one below that is the best fit for your research. If you are not sure, read the appropriate sections before making your selection.

\ Life sciences

Behavioural \& social sciences

Ecological, evolutionary \& environmental sciences

For a reference copy of the document with all sections, see nature.com/documents/nr-reporting-summary-flat.pdf

\section{Life sciences study design}

All studies must disclose on these points even when the disclosure is negative.

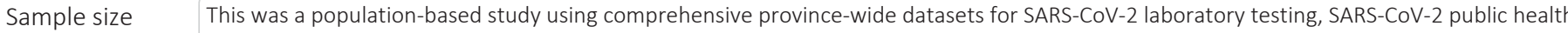
surveillance, COVID-19 vaccination, and healthcare system use employing test negative design study. As such, every individual tested for SARS-CoV-2 in Ontario and meeting study inclusion criteria were included in the study. No sample size was calculated. Sample size depended on the study outcome. For vaccine effectiveness against symptomatic infection, individuals who were symptomatic and tested positive for SARS-CoV-2 were considered as cases. For severe outcomes, test-positive individuals who had a hospitalization or death up to 17 August 2021 (regardless of the presence of any symptoms recorded at the time of RT-PCR testing) were considered as cases. Individuals who were symptomatic but only had tests negative for SARS-CoV-2 were considered as controls for both outcomes. Given that this was a populationbased study with only individuals who did not meet the eligibility criteria excluded, the sample sizes for each variant for both outcomes can be considered sufficient. Detailed sample sizes can be found in Supplementary Tables 1 and 2.

Data exclusions Exclusion criteria were specified a priori. Individuals who had tested positive for SARS-CoV-2 prior to their selected index date and those who received Ad26.COV2.S (Janssen), because it has not been used in Ontario (despite receiving approval), or mixed (ChAdOx1-mRNA or different mRNA) vaccine schedules were excluded. For severe outcomes, symptomatic test-negative individuals who later tested positive between 4 August 2021 and 17 August 2021 were excluded.

Replication Sensitivity analyses by estimating vaccine effectiveness $\geq 21$ days after the first dose for partial vaccination and $\geq 14$ days after the second dose for full vaccination, and restricting analyses to individuals who were tested between 5 April 2021 and 3 August 2021, all confirmed the results.

Randomization Not applicable as this is an observational study and the exposure (COVID-19 vaccine) was not randomly allocated.

Blinding Not applicable as this is an observational retrospective study and information on exposure and outcomes were extracted from comprehensive province-wide datasets.

\section{Reporting for specific materials, systems and methods}

We require information from authors about some types of materials, experimental systems and methods used in many studies. Here, indicate whether each material, system or method listed is relevant to your study. If you are not sure if a list item applies to your research, read the appropriate section before selecting a response.

\begin{tabular}{|c|c|c|c|}
\hline \multicolumn{2}{|c|}{ Materials \& experimental systems } & \multicolumn{2}{|c|}{ Methods } \\
\hline $\mathrm{n} / \mathrm{a}$ & Involved in the study & $\mathrm{n} / \mathrm{a}$ & Involved in the study \\
\hline$\bigotimes$ & $\square$ Antibodies & Х & ChIP-seq \\
\hline$\bigotimes$ & $\square$ Eukaryotic cell lines & Х & $\square$ Flow cytometry \\
\hline$\bigotimes$ & $\square$ Palaeontology and archaeology & Х & $\square$ MRI-based neuroimaging \\
\hline
\end{tabular}

\ $\square$ Animals and other organisms

$\square$ Human research participants

$\bigotimes \square$ Clinical data

$\bigotimes \square$ Dual use research of concern

\section{Human research participants}

Policy information about studies involving human research participants

Population characteristics

The characteristics of the study population for SARS-CoV-2 infection and severe outcomes can be found in Supplementary Table 1 and Supplementary Table 2, respectively.

Recruitment

This is a retrospective study where SARS-CoV-2 laboratory testing, SARS-CoV-2 public health surveillance, COVID-19 vaccination, healthcare system use, and related demographic information were extracted from linked comprehensive province-wide datasets. Study limitations related to potential biases and their likely impact have been discussed in the Discussion section.

Ethics oversight

ICES is a prescribed entity under Ontario's Personal Health Information Protection Act (PHIPA). Section 45 of PHIPA authorizes ICES to collect personal health information, without consent, for the purpose of analysis or compiling statistical 
information with respect to the management of, evaluation or monitoring of, the allocation of resources to or planning for all or part of the health system. Projects that use data collected by ICES under section 45 of PHIPA, and use no other data, are exempt from REB review. The use of the data in this project is authorized under section 45 and approved by ICES' Privacy and Legal Office.

Note that full information on the approval of the study protocol must also be provided in the manuscript. 